\title{
Author Index to Volume 24
}

\begin{tabular}{|c|c|}
\hline Äärimaa T, 745 & Chatburn RL, 217 \\
\hline Abrams SA, 693 & Chen LJ, 247 \\
\hline Abud R, 508 & Cheng E, 79 \\
\hline Adolph AL, 85 & Cherian MG, 326 \\
\hline Akabane T, 442 & Chesny RW, 657 \\
\hline Ammann AJ, 717 & Chevalier RL, 333 \\
\hline Amr S, 191 & Chitwood J, 145 \\
\hline Anceschi MM, 617 & Chowdhry P, 191 \\
\hline Anthony BF, 628 & Christensen $\mathrm{RD}, 605$ \\
\hline Antila $\mathrm{K}, 745$ & Chu SC, 243 \\
\hline Anwar M, 41 & Clemons G, 490 \\
\hline Aperia A, 46 & Coalson JJ, 357 \\
\hline Armstead W, 59 & Coble PA, 101 \\
\hline Assmann G, 222 & Cogneau M, 238 \\
\hline Auricchio RS, 233 & Cohen P; 25 \\
\hline Axelsson IE, 297 & $\begin{array}{l}\text { Colditz P, } 461 \\
\text { Collins JW, Jr, } 663\end{array}$ \\
\hline & $\begin{array}{l}\text { Collins JW, Jr, } 663 \\
\text { Coln D, } 438\end{array}$ \\
\hline Badoual J, 380 & Concepcion NF, 628 \\
\hline Barbati A, 617 & Constantopoulos GC, 347 \\
\hline Bard H, 470 & Cook CJ, 600 \\
\hline Barr SB, 735 & Cooper D, 353 \\
\hline Barrios NJ, 703 & Corbett RJT, 713 \\
\hline Bartolome J, 465, 583 & Corman N, 709 \\
\hline $\begin{array}{l}\text { Beasley DG, } 59 \\
\text { Beekman RH, } 186\end{array}$ & Cornell HJ, 233 \\
\hline $\begin{array}{l}\text { Beekman RH, } 186 \\
\text { Bell JM } 583\end{array}$ & Cosico L, 166,330 \\
\hline $\begin{array}{l}\text { Bell JM, } 583 \\
\text { Belli DC, } 34\end{array}$ & Cosmi EV, 617 \\
\hline $\begin{array}{l}\text { Belli DC, } 34 \\
\text { Ben Mansour A, } 751\end{array}$ & Cox KL, 243 \\
\hline $\begin{array}{l}\text { Ben Mansour A, } 751 \\
\text { Bennet L, } 600\end{array}$ & Crespo LM, 433 \\
\hline $\begin{array}{l}\text { Bennet L, } 600 \\
\text { Berni S, } 391\end{array}$ & Crissinger $\mathrm{KD}, 473$ \\
\hline Berry SA, 95 & \\
\hline Bifano EM, 433 & D'Allest AM, 477 \\
\hline Bisalli A, 751 & D'Ambola JB, 285 \\
\hline Black V, 63 & Dancis J, 63, 514 \\
\hline Blanco CE, 342 & Daniel SS, 73 \\
\hline Blizzard RM, 145 & Davis CB, 558 \\
\hline Blumer JL, 217 & Dawes GS, 342 \\
\hline Boccara JF, 380 & Day NL, 101 \\
\hline Bol A, 238 & De Castro G, 504 \\
\hline Bolhuis PA, 90 & de Escobar GM, 588 \\
\hline Bonnefont J-P, 308 & Dehan M, 477 \\
\hline Boucek MM, 499 & deLemos RA, 357 \\
\hline Boucher RC, 79 & del Rey FE, 588 \\
\hline Bowdy BD, 735 & Demaugre F, 308 \\
\hline Bowman FO Jr, 1 & Dembure P, 9 \\
\hline Bracci R, 391 & den Hollander JC, 622 \\
\hline Bradbury EM, 243 & de Oña CR, 588 \\
\hline Brann BS IV, 486 & De Ritis G, 233 \\
\hline Breider MA, 347 & Desjardins M, 6 \\
\hline Brook C, 145 & Desjeux J-F, 197, 751 \\
\hline Brownlee JR, 186 & Devaskar S, 683 \\
\hline Buckley S, 166, 171, 330 & De Vincenzi M, 233 \\
\hline Buescher ES, 14 & De Volder AG, 238 \\
\hline Buonocore G, 391 & Di Donato S, 308 \\
\hline Burchett SK, 717 & Diez de Pinos S, 740 \\
\hline Burr I, 145 & Dimand RJ, 243 \\
\hline Busch HMF, 90 & Di Renzo GC, 617 \\
\hline Busija D, 229 & Ducroc R, 197 \\
\hline Busija DW, 59 & Durand M, 203 \\
\hline Butkis A, 688 & Dussault JH, 6 \\
\hline Cairo MS, 673 & Eber $S, 213$ \\
\hline Cameron AM, 583 & Edwards BB, 20 \\
\hline Carey RM, 333 & Egan EA, 696 \\
\hline Carofilis A, 477 & Egan ML, 68 \\
\hline Casini A, 391 & Eklöf A-C, 46 \\
\hline Chalew S, 574 & El Monem AMA, 508 \\
\hline Chang HK, 203 & Elsas L, 9 \\
\hline Chang RL, 499 & English BK, 717 \\
\hline Chao G, 145 & English JD, 717 \\
\hline
\end{tabular}

Enns $\mathrm{G}, 166$

Ervin MG, 318

Esterman A, 63

Evans DH, 175

Evans HJ, 693

Fewell JE, 28

Finkel $Y, 46$

Finley SL, 384, 663

Fisher DA, 318

Fomon SJ, 20

Forbes D, 353

Fournier L-A, 34

Fouron J-C, 470

Francoual C, 380

Freedman JC, 433

Frenkel J, 622

Fujimoto T, 152

Furlanetto R, 145

Fyhrquist F, 490

Gahr M, 213

Galjaard H, 90

Gall DG, 353

Galli SA, 217

Garofano RP, 1

Garza C, 85, 693

Gaultier Cl, 477

Gelband H, 50

Gersony WM, 1

Ghishan FK, 338

Gibson KM, 455

Gibson N, 175

Gibson RL, 558

Gillespie MN, 735

Glomski CA, 703

Glorieux J, 6

Gluckman PD, 600

Goffinet AM, 238

Goldberg SJ, 740

Goldstein M, 486

Gomez RA, 333

Gong $\mathrm{H} \mathrm{Jr}, 285$

Granger DN, 473

Granoff DM, 180

Grant GP, 1

Grasset E, 197

Gray BM, 68

Greisen G, 461

Grose W, 644

Guenin K, 367

Guilleminault C, 477

Hallman M, 617

Hamosh M, 191

Hamosh P, 191

Hanson MA, 342

Hanukoglu A, 574

Harper RM, 677

Hashimoto T, 723

Hasui M, 442

Haven CA, 735

Hay WW Jr, 312

Hayde M, 222

Hayes CJ, 1

Hechtman P, 709

Heijden AJvd, 644

Heldt GP, 55

Hendrix MJC, 740

Henning SJ, 595
Herbst JJ, 247

Herin P, 423

Herschkowitz N, 367

Heyman M, 197

Ho L, 95

Hoffman HJ, 677

Holbrook ST, 605

Holman RT, 396

Holtzclaw L, 683

Holzinger T, 222

Hong JK, 628

Hop WCJ, 609

Horton JW, 438

Horvath A, 688

Hoshino Y, 160

Husain MK, 73

Iguchi $\mathrm{H}, 194$

Imura H, 668

Ito $\mathrm{E}, 668$

Ito $\mathrm{K}, 447$

Ito $S, 254$

Iwai S, 160

Iwai Y, 254

Jabs H-U, 222

Jaeken J, 238

Jakobsson I, 297

James LS, 73

James SP, 549

Janghorbani M, 20

Job JC, 380

Johnson SB, 396

Joiner $\mathrm{CH}, 495$

Kakehi T, 668

Kane RE, 247

Kashiwamata S, 209

Kato K, 194

Keino H, 209

Kerr DS, 95

Kikuchi K, 668

Kim K-M, 254

$\mathrm{Kim}$ KS, 628

Kjellmer I, 633

Kliegman RM, 302, 649

Klingensmith GJ, 145

Kluge KA, 677

Kobayashi Y, 442

Koen PA, 291

Kohno H, 194

Komiyama A, 442

Konduri GG, 28

Kosaki A, 668

Kowarski AA, 574

Krugman S, 512

Krystofik DA, 504

Kuehl TJ, 357

Kurland G, 243

Kuzuya H, 668

Kwun JE, 728

Lachowicz J, 465

Lam RW, 318

Lan C-C, 701

Larson JE, 156

Lau C, 583

Lazarow PB, 63

Leake RD, 318 
Lebenthal E, 508

Leblanc AD, 693

Lee P-C, 508

Leeper LL, 595

Leffler CW, 59, 229

Leonard D, 338

Leong $Y-m, 709$

Lepage $\mathrm{G}, 34$

Letarte J, 6

Levene MI, 175

Lewis MB, 322

Li C, 577

Liang D-C, 701

Lin-Chu M, 701

Linden JM, 333

Lindner W, 613

Lindquist BL, 508

Loeber CP, 740

Loonen MCB, 90

Lusk MM, 95

Ma S-W, 701

MacIsaac RJ, 688

Madsen K, 9

Mahony L, 639

Maiuri L, 233

Mansell AL, 1

Martha P, 145

Martinez AM, 427

Martinez JR, 427

Martini G, 391

Masuda M, 442

Matsuoka T, 442

Mayumi M, 254

McCooke HB, 342

McCormack J, 50

McDonald J, 145

McIlheran SM, 14

Merrick D, 663

Merrick JM, 508

Meznarich HK, 312

Michel Ch, 238

Mikawa H, 254, 668

Mirro R, 59

Misra R, 145

Mitchell G, 308

Miyabayashi S, 447

Mize CE, 713

Mock DM, 396

Mock NI, 396

Moonen CTW, 243

Mori M, 160

Morin FC, III, 696

Morissette J, 6

Morton S, 302

Moser A, 63

Moser H, 63

Murata K, 442

Murphy E, 79

Nagae H, 209

Naglak M, 9

Najjar J, 145

Narisawa K, 447

Natori S, 194

Nauta J, 644

Navarro HA, 465, 583
Nawata H, 194

Neijens HJ, 622

Neilson DW, 322

Nelson SE, 20

Nguyen-Hoang N, 308

Niida Y, 194

Nishikimi M, 447

Nishimura H, 668

Nogee LM, 568

Noordam MJ, 609

Norfleet WT, 696

Nunnally RL, 713

Nyhan WL, 455

Obara H, 160

Obregon MJ, 588

O'Brien JA, 728

Oda $Y, 668$

Odom MJ, 728

Ogata ES, 384, 663

Ogra PL, 504 ..

Oh W, 486

Oja R, 745

O'Loughlin EV, 353

Ong D, 481

Oranje WA, 644

Orenstein SR, 38

Orii T, 723

Ostedgaard LS, 20

Ottaviani MF, 391

Ozawa T, 447

Parsons HG, 353

Parton L, 166, 171, 330

Patel MS, 9.5

Pattarelli PP, 455

Pauly TH, 735

Peach MJ, 333

Pelet A, 308

Petrelli A, 617

Pezzoli S, 145

Phelps DL, 106

Picchi MP, 391

Pickoff AS, 50

Pietsch J, 338

Pontefract L, 688

Pourcyrous M, 229

Praud JP, 477

Prihoda TJ, 357

Pritchard DG, 68

Prosmanne J, 470

Provoost AP, 644

Pryds O, 461

Puri P, 152

Raia V, 233

Raivio KO, 373, 490

Ramet J, 477

Rautureau M, 751

Redding GJ, 558

Redha R, 481

Reen DJ, 152

Reuser AJJ, 90

Richardson GA, 101

Richter A, 709

Rihä NCR, 297

Rimoldi M, 308
Rivier J, 145

Rogers RR, 20

Rogol AD, 145

Rosenfeld CR, 728

Rosenthal A, 186

Ross MG, 318

Rothstein G, 605

Roy CC, 34

Rudolph AM, 577

Ruth V, 490

Saad M, 508

Sadiq F, 683

Said HM, 481

Saluna T, 166, 171, 330

Salvo R, 9

Santos M, 63

Saudubray J-M, 308

Sauer PJJ, 644

Schaad UB, 367

Schanler RJ, 693

Schaumberger M, 613

Schechtman VL, 677

Scher MS, 101

Schroeder R, 595

Seidler FJ, 583

Sender L, 673

Shackelford PG, 180

Shaddy RE, 577

Shaffer TH, 291

Sheng H-P, 85, 693

Sherman MP, 285

Shimomura Y, 447

Shimozawa N, 723g

Shinomiya K, 254

Shio H, 63

Shortland D, 175

Shull RM, 347

Silano V, 233

Sills RH, 703

Slotkin TA, 465, 583

Small GM, 63

Smith EO, 85

Smith P, 145

Snyder JM, 728

Sonesson S-E, 423

Southall DP, 677

Speer CP, 213

Stark RI, 73

Steffen C, 367

Stoffer DS, 101

Stolfi A, 50

Stonestreet BS, 486

Strober W, 549

Strobl W, 222

Stuart MJ, 563

Stutts MJ, 79

Sullivan KJ, 203

Sunderji S, 563

Suzuki H, 447

Suzuki Y, 723

Tada K, 447

Takayanagi N, 194

Talluri B, 391

Tamburlin JH, 703

Tanaka M, 254, 447
Tashkin DP, 285

Teitel DF, 577

Thaler MM, 247

Thieriot-Prevost G, 380

Thordstein M, 633

Thorner MO, 145

Thurlbeck WM, 156

Ting BTG, 20

Tischler D, 673

Tomé D, 751

Toy C, 673

Truog WE, 558

Tsukahara H, 668

Tyndall MR, 577

Uauy R, 713

Vale W, 145

Valimaki I, 745

Van den Berghe G, 238

van den Wijngaard JAGW, 609

Van der Ploeg AT, 90

Vandeven C, 673

van Dongen JJM, 622

Vannucci RC, 41

Versmold HT, 613

Vettenranta K, 373

Villafãne $\mathrm{J}, 50$

Volovitz B, 504

Walenga RW, 563

Wara DW, 717

Warburton D, 25, 166, 171, 330

Wass CA, 628

Watanabe K, 209

Weinberg GA, 180

Welliver RC, 504

Whitmore WL, 465

Widhalm K, 222

Wieland M, 213

Williams C, 600

Williamson SB, 713

Wilson AJ, 677

Wilson CB, 717

Wintour EM, 688

Wispe JR, 568

Witte MK, 217

Wladimiroff JW, 609

Wolff ED, 644

Wolfson MR, 291

Wolvers-Tettero ILM, 622

Xu H, 50

Yamada K, 668

Yamazaki M, 442

Yankaskas J, 79

Yasui K, 442

Ye T-H, 203

Yeagle PL, 703

Yokota S, 723

Yoshimasa Y, 668

Yousef IM, 34

Zaccardo G, 617

Ziegler EE, 20

Zlotkin SH, 326

Zubrow AB, 73 